\author{
K.A. Bekmaganbetov ${ }^{1}$, K.Ye. Kervenev ${ }^{2}$, Ye. Toleugazy ${ }^{1, *}$ \\ ${ }^{1}$ M.V. Lomonosov Moscow State University, Kazakhstan Branch, Nur-Sultan, Kazakhstan; \\ ${ }^{2}$ Karagandy University of the name of academician E.A. Buketov, Karaganda, Kazakhstan \\ (E-mail: bekmaganbetov-ka@yandex.kz,kervenev@bk.ru,toleugazy-y@yandex.ru)
}

\title{
The embedding theorems for anisotropic Nikol'skii-Besov spaces with generalized mixed smoothness
}

\begin{abstract}
The theory of embedding of spaces of differentiable functions studies the important relations of differential (smoothness) properties of functions in various metrics and has a wide application in the theory of boundary value problems of mathematical physics, approximation theory, and other fields of mathematics. In this article, we prove the embedding theorems for anisotropic spaces Nikol'skii-Besov with a generalized mixed smoothness and mixed metric, and anisotropic Lorentz spaces. The proofs of the obtained results are based on the inequality of different metrics for trigonometric polynomials in Lebesgue spaces with mixed metrics and interpolation properties of the corresponding spaces.
\end{abstract}

Keywords: anisotropic Lorentz spaces, anisotropic Nikol'skii-Besov spaces, generalized mixed smoothness, mixed metric, embedding theorems.

\section{Introduction}

One of the first results related to the theory of embedding spaces of differentiable functions belongs to S.L. Sobolev [1]. This theory studies important relations of differential (smoothness) properties of functions in various metrics. Further, the development of this theory is associated with new classes of function spaces defined and studied in the works of S.M. Nikol'skii [2, 3], O.V. Besov [4, 5], P.I. Lizorkin [6], H. Triebel [7, 8], and many others. The development of this research was determined both by its internal problems and by its applications in the theory of boundary value problems of mathematical physics and approximation theory (see, for example, $[9-14])$.

In this paper, embedding theorems for spaces with generalized mixed smoothness and with mixed metrics and anisotropic Lorentz spaces are obtained. The proofs of the achieved results are based on the inequalities of different metrics for trigonometric polynomials and interpolation theorems from the works of E.D. Nursultanov [15] and the authors [16].

\section{Definitions and auxiliary results}

Let $\mathbf{d}=\left(d_{1}, \ldots, d_{n}\right) \in \mathbb{N}^{n}, \mathbb{T}^{\mathbf{d}}=\left\{\mathbf{x}=\left(\mathbf{x}_{1}, \ldots, \mathbf{x}_{n}\right): \mathbf{x}_{i} \in \mathbb{T}^{d_{i}}=[0,2 \pi)^{d_{i}}, i=1, \ldots, n\right\}$ and $f(\mathbf{x})=f\left(\mathbf{x}_{1}, \ldots, \mathbf{x}_{n}\right)$ be measurable function on $\mathbb{T}^{\mathbf{d}}$.

Let $\mathbf{1} \leq \mathbf{p}=\left(p_{1}, \ldots, p_{n}\right) \leq \infty$. We say that the function $f$ belongs to the Lebesgue space with mixed metric $L_{\mathbf{p}}\left(\mathbb{T}^{\mathbf{d}}\right)$ if

$$
\|f\|_{L_{\mathbf{p}}\left(\mathbb{T}^{\mathbf{d}}\right)}=\left(\int_{\mathbb{T}^{d_{n}}}\left(\cdots\left(\int_{\mathbb{T}^{d_{1}}} \mid f\left(\mathbf{x}_{1}, \ldots,\left.\mathbf{x}_{n}\right|^{p_{1}} d \mathbf{x}_{1}\right)^{p_{2} / p_{1}} \ldots\right)^{p_{n} / p_{n-1}} d \mathbf{x}_{n}\right)^{1 / p_{n}}<\infty\right.
$$

In a case when $p_{i}=\infty$ the expression $\left(\int_{\mathbb{T}^{d_{i}}}\left|f\left(\mathbf{x}_{i}\right)\right|^{p_{i}} d \mathbf{x}_{i}\right)^{1 / p_{i}}$ means that ess $\sup _{\mathbf{x}_{i} \in \mathbb{T}^{d_{i}}}\left|f\left(\mathbf{x}_{i}\right)\right|$.

\footnotetext{
* Corresponding author.

E-mail: toleugazy-y@yandex.ru
} 
Let us denote by

$$
\Delta_{\mathbf{s}}(f, \mathbf{x})=\sum_{\mathbf{k} \in \rho(\mathbf{s})} a_{\mathbf{k}} e^{i\langle\mathbf{k}, \mathbf{x}\rangle_{\mathbf{d}}}
$$

the trigonometric series of $f \sim \sum_{\mathbf{k} \in \mathbb{Z}^{\mathbf{d}}} a_{\mathbf{k}} e^{i\langle\mathbf{k}, \mathbf{x}\rangle_{\mathbf{d}}}$, where $\langle\mathbf{k}, \mathbf{x}\rangle_{\mathbf{d}}=\sum_{i=1}^{n} \sum_{j=1}^{d_{i}} k_{j}^{i} x_{j}^{i}$ is the (modified) inner product, $\rho(\mathbf{s})=\left\{\mathbf{k}=\left(\mathbf{k}_{1}, \ldots, \mathbf{k}_{n}\right) \in \mathbb{Z}^{\mathbf{d}}:\left[2^{s_{i}-1}\right] \leq \max _{j=1, \ldots, d_{i}}\left|k_{j}^{i}\right|<2^{s_{i}}, i=1, \ldots, n\right\}$ and $[a]$ is the integer part of the number $a$.

Let $\alpha=\left(\alpha_{1}, \ldots, \alpha_{n}\right) \in \mathbb{R}^{n}, \mathbf{1} \leq \mathbf{q}=\left(\mathbf{q}_{\mathbf{1}}, \ldots, \mathbf{q}_{\mathbf{n}}\right) \leq \infty$ and $\mathbf{1}<\mathbf{p}=\left(p_{1}, \ldots, p_{n}\right)<\infty$.

The anisotropic Nikol'skii-Besov space with generalized mixed smoothness and mixed metric $B_{\mathbf{p}}^{\alpha \mathbf{q}}\left(\mathbb{T}^{\mathbf{d}}\right)$ is a set of the series $f \sim \sum_{\mathbf{k} \in \mathbb{Z}^{\mathbf{d}}} a_{\mathbf{k}} e^{i\langle\mathbf{k}, \mathbf{x}\rangle_{\mathbf{d}}}$ such that

$$
\|f\|_{B_{\mathbf{p}}^{\alpha \mathbf{q}}\left(\mathbb{T}^{\mathbf{d}}\right)}=\left\|\left\{2^{(\alpha, \mathbf{s})}\left\|\Delta_{\mathbf{s}}(f)\right\|_{L_{\mathbf{p}}\left(\mathbb{T}^{\mathbf{d}}\right)}\right\}\right\|_{l_{\mathbf{q}}}<\infty
$$

where $(\alpha, \mathbf{s})=\sum_{i=1}^{n} \alpha_{i} s_{i}$ is the inner product and $\|\cdot\|_{l_{\mathbf{q}}}$ is the norm of a discrete Lebesgue space with mixed metric $l_{\mathbf{q}}$.

Here $B_{\mathbf{p}}^{\alpha \mathbf{q}}\left(\mathbb{T}^{\mathbf{d}}\right)$ is a version of spaces, which was introduced and studied in [15].

Remark 1. The anisotropic Nikol'skii-Besov space with generalized mixed smoothness $B_{\mathbf{p}}^{\alpha \mathbf{q}}\left(\mathbb{T}^{\mathbf{d}}\right)$ mentioned above is a hybrid structure of Nikol'skii-Besov space (concerning variables included in one multi-variable) [2, 4] and spaces with dominant mixed derivative (concerning variables included in different multi-variables) [17, 18]. In the isotropic case, when $p$ and $q$ are scalars, analogs of these spaces were studied by D.B. Bazarkhanov [19].

Below we define an anisotropic interpolation method (see [20]) and interpolation theorems for Lebesgue spaces with mixed metric and anisotropic Nikol'skii-Besov spaces with generalized mixed smoothness.

Let $E=\left\{\varepsilon=\left(\varepsilon_{1}, \ldots, \varepsilon_{n}\right): \varepsilon_{i}=0\right.$ or $\left.\varepsilon_{i}=1, i=1, \ldots, n\right\}$ be vertices of the $n$-dimensional unit cube in $\mathbb{R}^{n}, \mathbf{A}=\left\{A_{\varepsilon}\right\}_{\varepsilon \in E}$ be compatible family of Banach spaces (this means that they are all embedded in a linear Hausdorff space). Let us define functional for $a \in \sum_{\varepsilon \in E} A_{\varepsilon}$

$$
K(\mathbf{t}, a ; \mathbf{A})=\inf _{a=\sum_{\varepsilon \in E}} \sum_{\varepsilon} \mathbf{t}^{\varepsilon}\left\|a_{\varepsilon}\right\|_{A_{\varepsilon}}
$$

here $t^{\varepsilon}=t_{1}^{\varepsilon_{1}} \cdot \ldots \cdot t_{n}^{\varepsilon_{n}}$

Moreover, for $\mathbf{0}<\theta=\left(\theta_{1}, \ldots, \theta_{n}\right)<\mathbf{1}, \mathbf{0}<\mathbf{r}=\left(r_{1}, \ldots, r_{n}\right) \leq \infty$, we denote by $\mathbf{A}_{\theta \mathbf{r}}=\left(A_{\varepsilon} ; \varepsilon \in E\right)_{\theta \mathbf{r}}$ the linear subset of the set $\sum_{\varepsilon \in E} A_{\varepsilon}$, such that

$$
=\left(\int_{0}^{\infty}\left(t_{n}^{-\theta_{n}} \ldots\left(\int_{0}^{\infty}\left(t_{1}^{-\theta_{1}} K(\mathbf{t}, a ; \mathbf{A})\right)^{r_{1}} \frac{d t_{1}}{t_{1}}\right)^{r_{2} / r_{1}} \ldots\right)^{r_{n} / r_{n-1}} \frac{d t_{n}}{t_{n}}\right)^{1 / r_{n}}<\infty .
$$

Lemma 1 ([20]). Let $\mathbf{0}<\theta<\mathbf{1}, \mathbf{0}<\mathbf{r} \leq \infty, \mathbf{A}=\left\{A_{\varepsilon}\right\}_{\varepsilon \in E}, \mathbf{B}=\left\{B_{\varepsilon}\right\}_{\varepsilon \in E}$ be two compatible families of Banach spaces. If there exist two vectors $\mathbf{M}_{\mathbf{0}}=\left(M_{1}^{0}, \ldots, M_{n}^{0}\right), \mathbf{M}_{\mathbf{1}}=\left(M_{1}^{1}, \ldots, M_{n}^{1}\right)$ with positive components such that for the linear operator $T$ the following mapping holds

$$
T: \mathbf{A}_{\varepsilon} \rightarrow \mathbf{B}_{\varepsilon}
$$

with the norm estimation $C_{\varepsilon} \prod_{i=1}^{n} M_{i}^{\varepsilon_{i}}$ for every $\varepsilon \in E$, then

$$
T: \mathbf{A}_{\theta \mathbf{r}} \rightarrow \mathbf{B}_{\theta \mathbf{r}}
$$

with the norm $\|T\|_{\mathbf{A}_{\theta \mathbf{r}} \rightarrow \mathbf{B}_{\theta \mathbf{r}}} \leq \max _{\varepsilon \in E} C_{\varepsilon} \prod_{i=1}^{n}\left(M_{i}^{0}\right)^{1-\theta_{i}}\left(M_{i}^{1}\right)^{\theta_{i}}$.

Let the multi-indices $\mathbf{p}=\left(p_{1}, \ldots, p_{n}\right), \mathbf{r}=\left(r_{1}, \ldots, r_{n}\right)$ be such that if $1 \leq p_{i}<\infty$, then $1 \leq r_{i} \leq \infty$, and if $p_{i}=\infty$, then $r_{i}=\infty(i=1, \ldots, n)$. 
The anisotropic Lorentz space $L_{\mathbf{p r}}\left(\mathbb{T}^{\mathbf{d}}\right)$ (see [15]) is a set of functions such that

$$
\begin{gathered}
\|f\|_{L_{\mathbf{p r}\left(\mathbb{T}^{\mathbf{d}}\right)}=} \\
=\left(\int_{0}^{(2 \pi)^{d_{n}}}\left(t_{n}^{1 / p_{n}} \ldots\left(\int_{0}^{(2 \pi)^{d_{1}}}\left(t_{1}^{1 / p_{1}} f^{*_{1}, \ldots, *_{n}}\left(t_{1}, \ldots, t_{n}\right)\right)^{r_{1}} \frac{d t_{1}}{t_{1}}\right)^{r_{2} / r_{1}} \ldots\right)^{r_{n} / r_{n-1}} \frac{d t_{n}}{t_{n}}\right)^{1 / r_{n}}<\infty,
\end{gathered}
$$

where $f^{*}(\mathbf{t})=f^{*_{1}, \ldots, *_{n}}\left(t_{1}, \ldots, t_{n}\right)$ is repeated non-increasing rearrangement of a function $f(\mathbf{x})=f\left(\mathbf{x}_{1}, \ldots, \mathbf{x}_{n}\right)$. In what follows by $f^{*}(\mathbf{t})=f^{*_{1}, \ldots, *_{n}}\left(t_{1}, \ldots, t_{n}\right)$ we mean the non-increasing rearrangement first with respect to $\mathbf{x}_{1}$ with fixed $\mathbf{x}_{2}, \ldots, \mathbf{x}_{n}$ and then with respect to $\mathbf{x}_{2}$ with fixed other (multi)variables and so on.

Let us denote $\mathbf{b}_{\varepsilon}=\left(b_{1}^{\varepsilon_{1}}, \ldots, b_{n}^{\varepsilon_{n}}\right)$ for multi-indices $\mathbf{b}_{0}=\left(b_{1}^{0}, \ldots, b_{n}^{0}\right), \mathbf{b}_{1}=\left(b_{1}^{1}, \ldots, b_{n}^{1}\right)$ and $\varepsilon=\left(\varepsilon_{1}, \ldots, \varepsilon_{n}\right) \in E$.

Lemma 2 ([15]). Let $\mathbf{1} \leq \mathbf{p}_{\mathbf{0}}=\left(p_{1}^{0}, \ldots, p_{n}^{0}\right)<\mathbf{p}_{\mathbf{1}}=\left(p_{1}^{1}, \ldots, p_{n}^{1}\right) \leq \infty$. Then for $\mathbf{0}<\theta=\left(\theta_{1}, \ldots, \theta_{n}\right)<\mathbf{1}$ and $\mathbf{1} \leq \mathbf{r}=\left(r_{1}, \ldots, r_{n}\right) \leq \infty$

$$
\left(L_{\mathbf{p}_{\varepsilon}}\left(\mathbb{T}^{\mathbf{d}}\right) ; \varepsilon \in E\right)_{\theta \mathbf{r}}=L_{\mathbf{p r}}\left(\mathbb{T}^{\mathbf{d}}\right),
$$

where $\mathbf{1} / \mathbf{p}=(\mathbf{1}-\theta) / \mathbf{p}_{\mathbf{0}}+\theta / \mathbf{p}_{\mathbf{1}}$.

Lemma 3 ([16]). Let $\mathbf{1}<\mathbf{p}=\left(p_{1}, \ldots, p_{n}\right)<\infty,-\infty<\alpha_{\mathbf{0}}=\left(\alpha_{1}^{0}, \ldots, \alpha_{n}^{0}\right)<\alpha_{\mathbf{1}}=\left(\alpha_{1}^{1}, \ldots, \alpha_{n}^{1}\right)<\infty$, $\mathbf{1} \leq \mathbf{q}_{\mathbf{0}}=\left(q_{1}^{0}, \ldots, q_{n}^{0}\right), \mathbf{q}_{\mathbf{1}}=\left(q_{1}^{1}, \ldots, q_{n}^{1}\right) \leq \infty, \varepsilon=\left(\varepsilon_{1}, \ldots, \varepsilon_{n}\right) \in E$. Then for $\mathbf{0}<\theta=\left(\theta_{1}, \ldots, \theta_{n}\right)<\mathbf{1}$ and $\mathbf{1} \leq \mathbf{q}=\left(q_{1}, \ldots, q_{n}\right) \leq \infty$

$$
\left(B_{\mathbf{p}}^{\alpha_{\varepsilon} \mathbf{q}_{\varepsilon}}\left(\mathbb{T}^{\mathbf{d}}\right) ; \varepsilon \in E\right)_{\theta \mathbf{q}}=B_{\mathbf{p}}^{\alpha \mathbf{q}}\left(\mathbb{T}^{\mathbf{d}}\right)
$$

here $\alpha=(1-\theta) \alpha_{0}+\theta \alpha_{1}$.

\section{Embedding theorems for anisotropic Nikol'skii-Besov spaces with generalized mixed smoothness}

In this section, the limit embedding theorems for different metrics for anisotropic Nikol'skii-Besov spaces with generalized mixed smoothness and anisotropic Lorentz spaces are proved.

Lemma 4 (Inequality of different metrics [2]). Let $T_{\mathbf{s}}(\mathbf{x})$ be trigonometric polynomial with the order no more than $\mathbf{s}=\left(s_{1}^{1}, \ldots, s_{d_{1}}^{1} ; \ldots ; s_{1}^{n}, \ldots, s_{d_{n}}^{n}\right)$ by multi-variable $\mathbf{x}=\left(x_{1}^{1}, \ldots, x_{d_{1}}^{1} ; \ldots ; x_{1}^{n}, \ldots, x_{d_{n}}^{n}\right)$. Then for $\mathbf{1}<\mathbf{p}_{\mathbf{1}}=$ $=\left(p_{1}^{1}, \ldots, p_{n}^{1}\right) \leq \mathbf{p}_{\mathbf{2}}=\left(p_{1}^{2}, \ldots, p_{n}^{2}\right)<\infty$

$$
\left\|T_{\mathbf{s}}\right\|_{L_{\mathbf{p}_{2}}\left(\mathbb{T}^{\mathbf{d}}\right)} \leq C \prod_{\left\{i: p_{i}^{1}<p_{i}^{2}\right\}} \prod_{j=1}^{d_{i}}\left(s_{j}^{i}\right)^{1 / p_{i}^{1}-1 / p_{i}^{2}}\left\|T_{\mathbf{s}}\right\|_{L_{\mathbf{p}_{1}}\left(\mathbb{T}^{\mathbf{d}}\right)},
$$

where $C$ is the positive constant which does not depend on $\mathbf{s}$.

Theorem 1. Let $-\infty<\alpha_{\mathbf{0}}=\left(\alpha_{1}^{0}, \ldots, \alpha_{n}^{0}\right) \leq \alpha_{\mathbf{1}}=\left(\alpha_{1}^{1}, \ldots, \alpha_{n}^{1}\right)<\infty, \mathbf{1} \leq \mathbf{q}=\left(q_{1}, \ldots, q_{n}\right) \leq \infty$ and $\mathbf{1}<\mathbf{p}_{\mathbf{0}}=\left(p_{1}^{0}, \ldots, p_{n}^{0}\right), \mathbf{p}_{\mathbf{1}}=\left(p_{1}^{1}, \ldots, p_{n}^{1}\right)<\infty$. Then the embedding

$$
B_{\mathbf{p}_{1}}^{\alpha_{1} \mathbf{q}}\left(\mathbb{T}^{\mathbf{d}}\right) \hookrightarrow B_{\mathbf{p o}_{\mathbf{0}}}^{\alpha_{\mathbf{0}} \mathbf{q}}\left(\mathbb{T}^{\mathbf{d}}\right)
$$

holds for $\alpha_{\mathbf{0}}-\mathbf{d} / \mathbf{p}_{\mathbf{0}}=\alpha_{\mathbf{1}}-\mathbf{d} / \mathbf{p}_{\mathbf{1}}$.

Proof. Let $f \in B_{\mathbf{p}_{\mathbf{1}}}^{\alpha_{1} \mathbf{q}}\left(\mathbb{T}^{\mathbf{d}}\right)$, then, according to the inequality of different metrics (Lemma 4), we obtain

$$
\begin{gathered}
\|f\|_{B_{\mathbf{p}_{\mathbf{0}} \mathbf{q}}^{\alpha_{\mathbf{q}}}}=\left\|\left\{2^{\left\langle\mathbb{T}_{\mathbf{0}}, \mathbf{s}\right\rangle}\left\|\Delta_{\mathbf{s}}(f)\right\|_{L_{\mathbf{p}_{\mathbf{0}}}\left(\mathbb{T}^{\mathbf{d}}\right)}\right\}\right\|_{l_{\mathbf{q}}} \leq \\
\leq C_{1}\left\|\left\{2^{\left\langle\alpha_{\mathbf{0}}+\mathbf{d}\left(\mathbf{1} / \mathbf{p}_{1}-\mathbf{1} / \mathbf{p}_{\mathbf{0}}\right), \mathbf{s}\right\rangle}\left\|\Delta_{\mathbf{s}}(f)\right\|_{L_{\mathbf{p}_{1}}\left(\mathbb{T}^{\mathbf{d}}\right)}\right\}\right\|_{l_{\mathbf{q}}}= \\
=C_{1}\left\|\left\{2^{\left\langle\alpha_{1}, \mathbf{s}\right\rangle}\left\|\Delta_{\mathbf{s}}(f)\right\|_{L_{\mathbf{p}_{1}}\left(\mathbb{T}^{\mathbf{d}}\right)}\right\}\right\|_{l_{\mathbf{q}}}=C_{1}\|f\|_{B_{\mathbf{p}_{1}}^{\alpha_{1} \mathbf{q}}\left(\mathbb{T}^{\mathbf{d}}\right)} .
\end{gathered}
$$

This completes the proof.

Theorem 2. Let $\mathbf{1}<\mathbf{p}=\left(p_{1}, \ldots, p_{n}\right)<\mathbf{q}=\left(q_{1}, \ldots, q_{n}\right)<\infty$ and $\mathbf{1} \leq \tau=\left(\tau_{1}, \ldots, \tau_{n}\right) \leq \infty$. Then the embedding

holds for $\alpha=(\mathbf{1} / \mathbf{p}-\mathbf{1} / \mathbf{q}) \mathbf{d}$.

$$
B_{\mathbf{p}}^{\alpha \tau}\left(\mathbb{T}^{\mathbf{d}}\right) \hookrightarrow L_{\mathbf{q} \tau}\left(\mathbb{T}^{\mathbf{d}}\right)
$$


Proof. According to the Minkowski inequality and the inequality of different metrics (Lemma 4), we receive

$$
\begin{gathered}
\|f\|_{L_{\mathbf{q}}\left(\mathbb{T}^{\mathbf{d}}\right)}=\left\|\sum_{\mathbf{k}=\mathbf{0}}^{\infty} \Delta_{\mathbf{k}}(f)\right\|_{L_{\mathbf{q}}\left(\mathbb{T}^{\mathbf{d}}\right)} \leq \\
\leq \sum_{\mathbf{k}=\mathbf{0}}^{\infty}\left\|\Delta_{\mathbf{k}}(f)\right\|_{L_{\mathbf{q}}\left(\mathbb{T}^{\mathbf{d}}\right)} \leq \sum_{\mathbf{k}=\mathbf{0}}^{\infty} 2^{((\mathbf{1} / \mathbf{p}-\mathbf{1} / \mathbf{q}) \mathbf{d}, \mathbf{k})}\left\|\Delta_{\mathbf{k}}(f)\right\|_{L_{\mathbf{p}}\left(\mathbb{T}^{\mathbf{d}}\right)}=\|f\|_{B_{\mathbf{p}}^{\alpha 1}\left(\mathbb{T}^{\mathbf{d}}\right)},
\end{gathered}
$$

where $\alpha=(\mathbf{1} / \mathbf{p}-\mathbf{1} / \mathbf{q}) \mathbf{d}$.

Therefore, for $\alpha=(\mathbf{1} / \mathbf{p}-\mathbf{1} / \mathbf{q}) \mathbf{d}$ we get

$$
B_{\mathbf{p}}^{\alpha 1}\left(\mathbb{T}^{\mathbf{d}}\right) \hookrightarrow L_{\mathbf{q}}\left(\mathbb{T}^{\mathbf{d}}\right) .
$$

Let us fix $\mathbf{p}=\left(p_{1}, \ldots, p_{n}\right)$ and let us choose $\alpha_{i}=\left(\alpha_{1}^{i}, \ldots, \alpha_{n}^{i}\right)$ and $\mathbf{q}_{i}=\left(q_{1}^{i}, \ldots, q_{n}^{i}\right)$ such that $\alpha_{j}^{i}=\left(1 / p_{j}-1 / q_{j}^{i}\right) d_{j}, i=0,1, j=1, \ldots, n$. Then for every $\varepsilon \in E$ we have

$$
B_{\mathbf{p}}^{\alpha_{\varepsilon} \mathbf{1}}\left(\mathbb{T}^{\mathbf{d}}\right) \hookrightarrow L_{\mathbf{q}_{\varepsilon}}\left(\mathbb{T}^{\mathbf{d}}\right) .
$$

According to Lemma 2 and Lemma 3, we obtain

$$
\left(B_{\mathbf{p}}^{\alpha_{\varepsilon} \mathbf{1}}\left(\mathbb{T}^{\mathbf{d}}\right) ; \varepsilon \in E\right)_{\theta \tau} \hookrightarrow\left(L_{\mathbf{q}_{\varepsilon} \mathbf{r}}\left(\mathbb{T}^{\mathbf{d}}\right) ; \varepsilon \in E\right)_{\theta \tau}
$$

or

$$
B_{\mathbf{p}}^{\alpha \tau}\left(\mathbb{T}^{\mathbf{d}}\right) \hookrightarrow L_{\mathbf{q} \tau}\left(\mathbb{T}^{\mathbf{d}}\right)
$$

where $\alpha=(\mathbf{1}-\theta) \alpha_{0}+\theta \alpha_{1}, \mathbf{1} / \mathbf{q}=(\mathbf{1}-\theta) / \mathbf{q}_{0}+\theta / \mathbf{q}_{1}$.

Let us check the relationship between the parameters $\alpha, \mathbf{p}$ and $\mathbf{q}$

$$
\begin{aligned}
\alpha & =(1-\theta) \alpha_{0}+\theta \alpha_{1}=(1-\theta)\left(\mathbf{1} / \mathbf{p}-\mathbf{1} / \mathbf{q}_{0}\right) \mathbf{d}+\theta\left(\mathbf{1} / \mathbf{p}-\mathbf{1} / \mathbf{q}_{1}\right) \mathbf{d}= \\
& =((1-\theta) / \mathbf{p}+\theta / \mathbf{p}) \mathbf{d}-\left((1-\theta) \mathbf{q}_{0}+\theta / \mathbf{q}_{1}\right) \mathbf{d}=(\mathbf{1} / \mathbf{p}-\mathbf{1} / \mathbf{q}) \mathbf{d} .
\end{aligned}
$$

The proof is complete.

Theorem 3. Let $\mathbf{1}<\mathbf{q}=\left(q_{1}, \ldots, q_{n}\right)<\mathbf{p}=\left(p_{1}, \ldots, p_{n}\right)<\infty$ and $\mathbf{1} \leq \tau=\left(\tau_{1}, \ldots, \tau_{n}\right) \leq \infty$. Then the embedding

$$
L_{\mathbf{q} \tau}\left(\mathbb{T}^{\mathbf{d}}\right) \hookrightarrow B_{\mathbf{p}}^{\alpha \tau}\left(\mathbb{T}^{\mathbf{d}}\right)
$$

holds for $\alpha=(\mathbf{1} / \mathbf{p}-\mathbf{1} / \mathbf{q}) \mathbf{d}$.

Proof. According to the inequality of different metrics (Lemma 4) and M. Riesz's theorem on the boundedness of parallelepiped partial sums, we obtain

$$
\begin{gathered}
\|f\|_{B_{\mathbf{p}}^{\alpha \infty}\left(\mathbb{T}^{\mathbf{d}}\right)}=\sup _{\mathbf{k} \geq \mathbf{0}} 2^{(\alpha, \mathbf{k})}\left\|\Delta_{\mathbf{k}}(f)\right\|_{L_{\mathbf{p}}\left(\mathbb{T}^{\mathbf{d}}\right)} \leq \\
\leq C_{1} \sup _{\mathbf{k} \geq \mathbf{0}} 2^{(\alpha+(\mathbf{1} / \mathbf{q}-\mathbf{1} / \mathbf{p}) \mathbf{d}, \mathbf{k})}\left\|\Delta_{\mathbf{k}}(f)\right\|_{L_{\mathbf{q}}\left(\mathbb{T}^{\mathbf{d}}\right)}=C_{1} \sup _{\mathbf{k} \geq \mathbf{0}}\left\|\Delta_{\mathbf{k}}(f)\right\|_{L_{\mathbf{q}\left(\mathbb{T}^{\mathbf{d}}\right)}} \leq C_{2}\|f\|_{L_{\mathbf{q}}\left(\mathbb{T}^{\mathbf{d}}\right)},
\end{gathered}
$$

since $\alpha=(\mathbf{1} / \mathbf{p}-\mathbf{1} / \mathbf{q}) \mathbf{d}$.

Thus, for $\alpha=(\mathbf{1} / \mathbf{p}-\mathbf{1} / \mathbf{q}) \mathbf{d}$ we have

$$
L_{\mathbf{q}}\left(\mathbb{T}^{\mathbf{d}}\right) \hookrightarrow B_{\mathbf{p}}^{\alpha \infty}\left(\mathbb{T}^{\mathbf{d}}\right) .
$$

Let us fix $\mathbf{p}=\left(p_{1}, \ldots, p_{n}\right)$ and let us choose parameters $\alpha_{i}=\left(\alpha_{1}^{i}, \ldots, \alpha_{n}^{i}\right)$ and $\mathbf{q}_{i}=\left(q_{1}^{i}, \ldots, q_{n}^{i}\right)$ such that $\alpha_{j}^{i}=\left(1 / p_{j}-1 / q_{j}^{i}\right) d_{j}, i=0,1, j=1, \ldots, n$. Then for every $\varepsilon \in E$ we receive

$$
L_{\mathbf{q}_{\varepsilon}}\left(\mathbb{T}^{\mathbf{d}}\right) \hookrightarrow B_{\mathbf{p}}^{\alpha_{\varepsilon} \infty}\left(\mathbb{T}^{\mathbf{d}}\right) .
$$

According to Lemma 2 and Lemma 3 we obtain

$$
\left(L_{\mathbf{q}_{\varepsilon}}\left(\mathbb{T}^{\mathbf{d}}\right) ; \varepsilon \in E\right)_{\theta \tau} \hookrightarrow\left(B_{\mathbf{p}}^{\alpha_{\varepsilon} \infty}\left(\mathbb{T}^{\mathbf{d}}\right) ; \varepsilon \in E\right)_{\theta \tau}
$$


or

$$
L_{\mathbf{q} \tau}\left(\mathbb{T}^{\mathbf{d}}\right) \hookrightarrow B_{\mathbf{p}}^{\alpha \tau}\left(\mathbb{T}^{\mathbf{d}}\right)
$$

where $\alpha=(\mathbf{1}-\theta) \alpha_{0}+\theta \alpha_{1}, \mathbf{1} / \mathbf{q}=(\mathbf{1}-\theta) / \mathbf{q}_{0}+\theta / \mathbf{q}_{1}$.

Let us check the relationship between the parameters $\alpha, \mathbf{p}$ and $\mathbf{q}$

$$
\begin{aligned}
\alpha & =(1-\theta) \alpha_{0}+\theta \alpha_{1}=(1-\theta)\left(\mathbf{1} / \mathbf{p}-\mathbf{1} / \mathbf{q}_{0}\right) \mathbf{d}+\theta\left(\mathbf{1} / \mathbf{p}-\mathbf{1} / \mathbf{q}_{1}\right) \mathbf{d}= \\
& =((\mathbf{1}-\theta) / \mathbf{p}+\theta / \mathbf{p}) \mathbf{d}-\left((\mathbf{1}-\theta) / \mathbf{q}_{0}+\theta / \mathbf{q}_{1}\right) \mathbf{d}=(\mathbf{1} / \mathbf{p}-\mathbf{1} / \mathbf{q}) \mathbf{d}
\end{aligned}
$$

The proof is complete.

Remark 2. 1) It is possible to show that the conditions of Theorems $1-3$ are unimprovable. The proof of these facts can be carried out by analogy with the corresponding proof from the paper [21]. $[15]$.

2 ) In a case when $\mathbf{d}=(1, \ldots, 1)$ the results of Theorems $1-3$ were announced in the paper by E.D. Nursultanov

\section{Acknowledgments}

The research was partially supported by the grant of the Science Committee of the Ministry of Education and Science of the Republic of Kazakhstan (Grant AP08855579).

\section{References}

1 Sobolev, S.L. (1938). On a theorem of functional analysis. Amer. Math. Soc. Transl., Ser. 2, 34, 39-68.

2 Nikol'skii, S.M. (1969). Inequalities for entire functions of finite degree and their application to the theory of differentiable functions of several variables. Amer. Math. Soc. Transl., Ser. 2, 80, 1-38.

3 Nikol'skii, S.M. (1975). Approximation of functions of several variables and imbedding theorems. New York-Heidelberg: Springer-Verlag.

4 Besov, O.V. (1964). Investigation of a family of function spaces in connection with theorems of imbedding and extension. Amer. Math. Soc. Transl., Ser. 2, 40, 85-126.

5 Besov, O.V., Il'in, V.P., \& Nikol'skii, S.M. Integral representations of functions and imbedding theorems. Washington: Winston; New York-Toronto-London: Wiley.

6 Lizorkin, P.I. (1962). $L_{p}^{r}(\Omega)$ spaces. Extension and imbedding theorems. Soviet Math. Dokl., 3, $1053-1057$.

7 Triebel, H. (1973). Spaces of distributions of Besov type on Euclidean $n$-space. Duality, interpolation. Ark. Mat., $11(1-2), 13-64$.

8 Triebel, H. (1978). Theory of interpolation. Functional spaces. Differential operators. Berlin: VEB Deutscher Verlag der Wissenschaften.

9 Bekmaganbetov, K.A., Chechkin, G.A., \& Chepyzhov, V.V. (2016). Homogenization of random attractors for reaction-diffusion systems. CR Mecanique, 344, 753-758.

10 Bekmaganbetov, K.A., Chechkin, G.A., Chepyzhov, V.V., \& Goritsky, A.Yu. (2017). Homogenization of trajectory attractors of 3D Navier-Stokes system with randomly oscillating force. Discrete Contin. Dyn. Syst., 37, 2375-2393.

11 Nursultanov, E.D., \& Tleukhanova, N.T. (2003). Quadrature formulae for classes of functions of low smoothness. Sbornik Math., 194(10), 1559-1584.

12 Akishev, G.A. (2006). Approximation of function classes in spaces with mixed norm. Sbornik Math., $197(7-8), 1121-1144$.

13 Bekmaganbetov, K.A., \& Toleugazy, Y. (2016). Order of the orthoprojection widths of the anisotropic Nikol'skii-Besov classes in the anisotropic Lorentz space. Eurasian Mathematical Journal, 7(3), 8-16.

14 Bekmaganbetov, K.A., \& Toleugazy, Y. (2019). On the order of the trigonometric diameter of the anisotropic Nikol'skii-Besov class in the metric anisotropic Lorentz spaces. Analysis Mathematica, 45 (2), $237-247$.

15 Nursultanov, E.D. (2004). Interpolation theorems for anisotropic function spaces and their applications. Dokl. Math., 69(1), 16-19. 
16 Bekmaganbetov, K.A., Kervenev, K.Ye., \& Toleugazy, Ye. (2020). Interpolation theorem for Nikol'skiiBesov type spaces with mixed metric. Bulletin of the Karaganda University. Mathematics series, 100(4), $33-42$.

17 Dzhabrailov, A.D. (1964). On some function spaces. Direct and inverse imbedding theorems. Abstract os Papers of Nauka SSSR, 159, 254-257.

18 Amanov, T.I. (1965). Representation and imbedding theorems for the function spaces $S_{p, \theta}^{(r)} B\left(R_{n}\right)$ and $S_{p^{*}, \theta}^{(r)} B\left(0 \leq x_{j} \leq 2 \pi ; j=1, \ldots, n\right)$. Proc. Steklov Inst. Math., $77,3-36$.

19 Bazarkhanov, D.B. (2005). Equivalent (quasi)norms for certain function spaces of generalized mixed smoothness. Proc. Steklov Inst. Math., 248, 21-34.

20 Nursultanov, E.D. (2000). On the coefficients of multiple Fourier series in $L_{p}$-spaces. Izvestia Mathematics, $64(1), 93-120$.

21 Bekmaganbetov, K.A., \& Nursultanov, E.D. (2009). Embedding theorems for anisotropic Besov spaces $B_{\mathbf{p r}}^{\alpha \mathbf{q}}\left([0 ; 2 \pi)^{n}\right)$. Izvestia Mathematics,, 73(4), 655-668.

\author{
Қ.А. Бекмағанбетов ${ }^{1}$, Қ.Е. Кервенев ${ }^{2}$, Е. Төлеуғазы ${ }^{1}$ \\ ${ }^{1}$ М.В. Ломоносов атындазы Мәскеу мемлекеттік университеті, \\ Қазақстан филиалы, Нұр-Сұлтан, Қазақстан; \\ ${ }^{2}$ Академик Е.А. Бөкетов атындавы Қараганды университеті, Қараванды, Қазақстан
}

\title{
Жалпыланған аралас тегістілігі бар Никольский-Бесовтың анизотропты кеңістіктері үшін енгізу теоремалары
}

\begin{abstract}
Дифференциалданатын функциялар кеңістіктерінің енгізу теориясы әртүрлі метрикалардағы функциялардың дифференциалдық (тегістіліктік) қасиеттерінің маңызды байланыстары мен қатынастарын зерттейді. Математикалық физиканың шектік есептер теориясында, жуықтау теориясында және математиканың басқа да салаларында кеңінен колданысқа ие. Мақалада жалпыланған аралас тегістілігі және аралас метрикасы бар Никольский-Бесовтың анизотропты кеңістіктері үшін және Лоренцтің анизотропты кеңістіктері үшін енгізу теоремалары дәлелдеген. Алынған нәтижелердің дәлелдеулері аралас метрикасы бар Лебег кеңістіктеріндегі тригонометриялық полиномдар үшін әртүрлі метрикалар теңсіздіктерін және сәйкес кеңістіктердің интерполяциялық қасиеттерін қолдануға негізделген.
\end{abstract}

Kiлm сөздер: Лоренцтің анизотропты кеңістіктері, Никольский-Бесовтың анизотропты кеңістіктері, жалпыланған аралас тегістілік, аралас метрика, енгізу теоремалары.

\author{
K.А. Бекмаганбетов ${ }^{1}$, K.Е. Кервенев ${ }^{2}$, Е. Толеугазы ${ }^{1}$ \\ ${ }^{1}$ Московский государственный университет имени М.В. Ломономова, \\ Казахстанский филиал, Нур-Султан, Казахстан; \\ ${ }^{2}$ Карагандинский университет имени академика Е.А. Букетова, Караганда, Казахстан
}

\section{Теоремы вложения для анизотропных пространств Никольского-Бесова с обобщенной смешанной гладкостью}

Теория вложения пространств дифференцируемых функций изучает важные связи и соотношения дифференциальных (гладкостных) свойств функций в различных метриках и имеет широкое применение в теории краевых задач математической физики, теории приближений и других разделах математики. В статье авторами доказаны теоремы вложения для анизотропных пространств НикольскогоБесова с обобщенной смешанной гладкостью, со смешанной метрикой и для анизотропных пространств Лоренца. Доказательства полученных результатов основаны на использовании неравенства 
разных метрик для тригонометрических полиномов в пространствах Лебега со смешанной метрикой и интерполяционных свойствах соответствующих пространств.

Ключевые слова: анизотропные пространства Лоренца, анизотропные пространства НикольскогоБесова, обобщенная смешанная гладкость, смешанная метрика, теоремы вложения. 